\title{
Report on Astronomical Constants
}

\author{
Toshio Fukushima, Chair, IAU WG on Astronomical Standards \\ National Astronomical Observatory, Mitaka, Tokyo, Japan
}

\begin{abstract}
Given was additional information to a previous report on the recent progress in the determinations of astronomical constants (Fukushima 2000), including the revision of $L_{G}, \Delta p$ and the offsets of CEP. As a summary, presented was the (revised) IAU 2000 File of Current Best Estimates of astronomical constants, which is to replace the former 1994 version (Standish 1995).
\end{abstract}

\section{Introduction}

The report of the IAU Working Group on Astronomical Standards (WGAS) on the issue of astronomical constants has appeared already (Fukushima 2000). Since its publication, made were only a few revisions, whose details will be reported here. One is a minor correction of the numerical value of $L_{G}$, which was to be consistent with the other proposals submitted simultaneously (Petit 2000). Another is the revision of the correction to the precession constant based on the very recent re-analysis of LLR observations (Chapront et al. 2000).

We admit that a misprint in the value of $L_{G}$ appeared in Fukushima (2000). The correct value (Petit 2000), which has been adopted in the IAU 2000 Resolution $\mathrm{B} 1.9$, is

$$
L_{G}=6.969290134 \times 10^{-10}
$$

which has no uncertainty. Or, more rigorously speaking, it is no more a primary constant determined from measurements but has become a defining constant, the constant defining the TCG. Thus, by combining its value with the recent determination of $L_{C}$ (see Fukushima 2000) as

$$
L_{C}=1.4808268674 \times 10^{-8} \pm 1.4 \times 10^{-17},
$$

we now have a new estimate of another scale constant, $L_{B}$, as

$$
L_{B}=1.5505197677 \times 10^{-8} \pm 2.0 \times 10^{-17}
$$

which is to be used to convert the (obsolete) TDB-based determinations to the TCB based ones. As an application, we computed the TCB-based value of $G M_{E}$ from that given in Groten (2000) as

$$
G M_{E}=3.9860044415 \times 10^{14} \pm 8 \times 10^{5} \mathrm{~m}^{3} \mathrm{~s}^{-2}
$$




\section{Precession Constant}

As was already stated, we can obtain a more reliable value for the correction to the precession constant, which was recently derived by the LLR observation (Chapront et al. 2000). Then, we expanded the table showing the history of $\Delta p$ determination given in Fukushima (2000) and listed it here as Table 1. Note that, in the references of this article, we have listed only those whose informations were not available before the publication of the previous work (Fukushima 2000). The latest LLR-based determination (Chapront et al. 2000) of $\Delta p$ was close to but clearly different from the VLBI-based ones as

$$
\Delta^{(\mathrm{L})} p=(-0.3164 \pm 0.0030)^{\prime \prime} / \mathrm{cy} .
$$

On the other hand, the VLBI-based best estimate (Fukushima 2000) was

$$
\Delta^{(\mathrm{V})} p=(-0.2968 \pm 0.0043)^{\prime \prime} / \mathrm{cy},
$$

Unfortunately, there is still a significant difference between these two determinations. We simply take their mean to derive the best estimate of $\Delta p$ as

$$
\Delta p=(-0.3066 \pm 0.0048)^{\prime \prime} / \text { cy. }
$$

By adding this to the IAU 1976 value of precession constant, we now have the best estimate of the general precession in longitude as

$$
p=\left(\begin{array}{ll}
5 & 028.790 \pm 0.005
\end{array}\right)^{\prime \prime} / \mathrm{cy} .
$$

At the same time, the recent estimates of the offset of Celestial Ephemeris Pole at the epoch J2000.0, $\Delta \psi_{0} \sin \varepsilon_{0}$ and $\Delta \varepsilon_{0}$, seem to converge to a single pair of values being independent on the observation type. See Table 2 .

By adopting a similar procedure as we did in deriving $\Delta p$, we obtained the offsets as

$$
\begin{aligned}
& \Delta^{(\mathrm{V})} \psi_{0} \sin \varepsilon_{0}=(-16.7 \pm 0.5) \mathrm{mas}, \\
& \Delta^{(\mathrm{V})} \varepsilon_{0}=(-4.9 \pm 0.3) \mathrm{mas}, \\
& \Delta^{(\mathrm{L})} \psi_{0} \sin \varepsilon_{0}=(-17.3 \pm 0.4) \mathrm{mas}, \Delta^{(\mathrm{L})} \varepsilon_{0}=(-5.4 \pm 0.2) \mathrm{mas}, \\
& \Delta \psi_{0} \sin \varepsilon_{0}=(-17.0 \pm 0.3) \mathrm{mas}, \Delta \varepsilon_{0}=(-5.2 \pm 0.3) \mathrm{mas},
\end{aligned}
$$

Note that $\Delta \varepsilon_{0}$ thus obtained is the correction not to the IAU 1976 value,

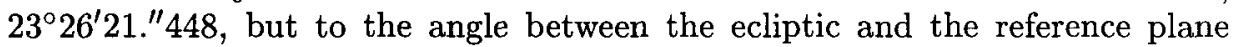
of International Celestial Reference System (ICRS). Quoting the result of investigation of Chapront et al. (1999), where the obliquity of the inertial mean ecliptic to the ICRS equator was estimated as

$$
\varepsilon_{0}(\text { ICRS })=23^{\circ} 26^{\prime} 21 . .^{\prime \prime} 41100 \pm 0 .^{\prime \prime} 00005,
$$

we now have the best estimate of the obliquity of the ecliptic at J2000.0 as

$$
\varepsilon_{0}=23^{\circ} 26^{\prime} 21 . " 4059 \pm 0 .{ }^{\prime \prime} 0003 .
$$

This is significantly different from the value of JPL's DE series, $23^{\circ} 26^{\prime} 21 . " 412$. 
Table 1. Corrections to Precession Constants

\begin{tabular}{llllll}
\hline Method \& Reference & \multicolumn{3}{c}{$\Delta p($ "Ify) } & \multicolumn{2}{c}{$\Delta \varepsilon_{1}($ (")/cy) } \\
& & Value & $\sigma$ & Value & $\sigma$ \\
\hline V & Fanselow et al. (1984) & -0.38 & 0.09 & & \\
V & Herring et al. (1986) & -0.239 & 0.013 & & \\
V & Zhu et al. (1990) & -0.38 & 0.05 & +0.017 & 0.017 \\
V & Sovers (1990) & -0.196 & 0.013 & & \\
S & Andrei \& Elsmore (1991) & +0.01 & 0.15 & & \\
V & Herring et al. (1991) & -0.32 & 0.10 & -0.04 & 0.05 \\
L+V & Williams et al. (1991) & -0.27 & 0.04 & & \\
V & McCarthy \& Luzum (1991) & -0.27 & 0.02 & -0.005 & 0.007 \\
P & Miyamoto \& Soma (1993) & -0.27 & 0.03 & & \\
V & Walter \& Ma (1994) & -0.36 & 0.11 & & \\
T & Williams (1994) & -0.2368 & & -0.0244 & \\
L+V & Charlot et al. (1995) & -0.30 & 0.02 & -0.020 & 0.008 \\
V & Herring (1995) & -0.30 & 0.01 & -0.024 & 0.005 \\
V & Souchay et al. (1995) & -0.321 & 0.003 & -0.026 & 0.001 \\
V & Walter \& Sovers (1996) & -0.31 & 0.01 & & \\
O & Vondrák (1999) & -0.154 & 0.004 & -0.013 & 0.0018 \\
L & Chapront et al. (1999) & -0.344 & 0.004 & & \\
P & Vityazev (2000) & -0.28 & 0.08 & & \\
O & Vondrák and Ron (2000) & -0.216 & 0.005 & -0.0093 & 0.0018 \\
V & Petrov (2000) & -0.295 & 0.002 & -0.027 & 0.0009 \\
V & Vondrák and Ron (2000) & -0.2990 & 0.0013 & -0.022 & 0.0007 \\
V & Mathews et al. (2000) & -0.3001 & 0.0008 & -0.0247 & 0.0003 \\
V & Shirai \& Fukushima (2000) & -0.2930 & 0.0005 & -0.0243 & 0.0002 \\
L & Chapront et al. (2000) & -0.3164 & 0.0030 & & \\
\hline
\end{tabular}

Note: The symbols of the methods are; V for the VLBI data, $\mathrm{S}$ for the short baseline radio interferometry, $L$ for the LLR data, $P$ for the proper motion analysis, $T$ for the theoretical consideration, and $O$ for the optical observation of latitude variations.

Table 2. Offsets of Celestial Ephemeris Pole at J2000.0

\begin{tabular}{llllll}
\hline Method \& Reference & \multicolumn{2}{c}{$\Delta \psi_{0} \sin \varepsilon_{0}$ (mas) } & \multicolumn{2}{c}{$\Delta \varepsilon_{0}$ (mas) } \\
& Value & $\sigma$ & Value & $\sigma$ \\
\hline V & Herring (1995) & -17.3 & 0.2 & -5.1 & 0.2 \\
L & Chapront et al. (1999) & -18.3 & 0.4 & -5.6 & 0.2 \\
O & Vondrák \& Ron (2000) & -12.3 & 0.7 & -9.2 & 0.6 \\
V & Vondrák \& Ron (2000) & -17.10 & 0.05 & -4.95 & 0.05 \\
V & Mathews et al. (2000) & -16.18 & & -4.53 & \\
V & Shirai \& Fukushima (2000) & -16.889 & 0.013 & -5.186 & 0.013 \\
L & Chapront et al. (2000) & -17.3 & 0.4 & -5.4 & 0.2 \\
\hline
\end{tabular}


Table 3. IAU 2000 File of Current Best Estimates

\begin{tabular}{lll}
\hline Class \& Item & Value (Uncertainty) [Unit] & Reference \\
\hline DEFINING & & \\
$k$ & $1.720209895 \times 10^{-2}$ & IAU 1976 \\
$c$ & $2.99792458 \times 10^{8}\left[\mathrm{~ms}^{-1}\right]$ & CODATA 1998 \\
$L_{\mathrm{G}}$ & $6.969290134 \times 10^{-10}$ & Petit (2000) \\
PRIMARY & & \\
$L_{\mathrm{C}}$ & $1.4808268674(14) \times 10^{-8}$ & Irwin and \\
& & Fukushima (1999) \\
$p$ & $5.028790(5) \times 10^{3}\left[{ }^{\prime \prime} / \mathrm{cy}\right]$ & This article \\
$\varepsilon_{0}$ & $8.43814059(3) \times 10^{4}\left[{ }^{\prime \prime}\right]$ & This article \\
$\tau_{\mathrm{A}}$ & $4.9900478639(2) \times 10^{2}[\mathrm{~s}]$ & DE405 \\
$M_{\mathrm{M}} / M_{\mathrm{E}}$ & $1.23000345(5) \times 10^{-2}$ & DE405 \\
$M_{\mathrm{S}} / M_{\mathrm{Me}}$ & $6.0236(3) \times 10^{6}$ & Andersen et al. $(1987)$ \\
$M_{\mathrm{S}} / M_{\mathrm{V}}$ & $4.0852371(6) \times 10^{5}$ & Sjogren et al. $(1990)$ \\
$M_{\mathrm{S}} / M_{\mathrm{Ma}}$ & $3.098708(9) \times 10^{6}$ & Null (1969) \\
$M_{\mathrm{S}} / M_{\mathrm{J}}$ & $1.0473486(8) \times 10^{3}$ & Campbell and \\
& & Synott (1985) \\
$M_{\mathrm{S}} / M_{\mathrm{Sa}}$ & $3.497898(18) \times 10^{3}$ & Campbell and \\
& & Anderson $(1989)$ \\
$M_{\mathrm{S}} / M_{\mathrm{U}}$ & $2.290298(3) \times 10^{4}$ & Jacobson et al. $(1992)$ \\
$M_{\mathrm{S}} / M_{\mathrm{N}}$ & $1.941224(4) \times 10^{4}$ & Jacobson et al. $(1991)$ \\
$M_{\mathrm{S}} / M_{\mathrm{P}}$ & $1.3521(15) \times 10^{8}$ & Tholen and \\
& & Buie (1997) \\
$a_{\mathrm{E}}$ & $6.3781366(1) \times 10^{6}[\mathrm{~m}]$ & Groten $(2000)$ \\
$J_{2}$ & $1.0826359(1) \times 10^{-3}$ & Groten (2000) \\
$G M_{\mathrm{E}}$ & $3.986004415(8) \times 10^{14}\left[\mathrm{~m}^{3} \mathrm{~s}^{-2}\right]$ & This article \\
$W_{0}$ & $6.26368560(5) \times 10^{7}\left[\mathrm{~m}^{2} \mathrm{~s}^{-2}\right]$ & Groten $(2000)$ \\
$\omega$ & $7.2921150(1) \times 10^{-11}\left[\mathrm{rad} \mathrm{s}^{-1}\right]$ & Groten (2000) \\
$G$ & $6.673(10) \times 10^{-11}\left[\mathrm{~m}^{3} \mathrm{~kg}^{-1} \mathrm{~s}^{-2}\right]$ & CODATA 1998 \\
\hline
\end{tabular}

Note: The exponent expressions are fully introduced. The units of uncertainties are the last digit of the values shown. The value of $\tau_{\mathrm{A}}$ shown here is that after the scale transformation was applied. The value before transformation, originally given DE405, is $499.0047838061 \ldots$ The geophysical vilues are those for the tide free system (Groten 2000). Suffices of radii and masses indicate the celestial objects; $E$ for the Earth, $M$ for the Moon, S for the Sun, Me for Mercury, V for Venus, Ma for Mars, J for Jupiter, $\mathrm{Sa}$ for Saturn, U for Uranus, $\mathrm{N}$ for Neptune, and $\mathrm{P}$ for Pluto. Note that the planetary masses except the Earth include the contribution of their satellites. 


\section{Conclusion}

By collecting the results on the two topics described in the previous sections, we updated the former IAU File of Current Best Estimates of astronomical constants (Standish 1995). The revised list is illustrated in Table 2.. Here the references for the items differring from the previous version (Standish 1995) are: (1) Tholen and Buie (1997) for the mass ratio of Pluto+Charon to that of the Sun, $M_{\mathrm{S}} / M_{\mathrm{P}},(2) \mathrm{DE} 405$ for $\tau_{\mathrm{A}}$ and $M_{\mathrm{M}} / M_{\mathrm{E}}$, (3) IAG 1999 for the geodetic constants, $a_{\mathrm{E}}, J_{2}, 1 / f$, and $W_{0},(4)$ CODATA 1998 for $G$, (5) Petit (2000) for $L_{G}$, and (6) this article for $G M_{E}, p$, and $\varepsilon_{0}$.

\section{References}

Anderson, J.D., Colombo, G., Esposito, P.B., Lau, E.L., \& Trager, G.B. 1987, Icarus, 71,337

Andrei, A.H., \& Elsmore, B. 1991, Proc. IAU Colloq. 127, 157

Campbell, J.K., \& Anderson, J.D. 1989, AJ, 97, 1485.

Campbell, J.K., \& Synott, S.P. 1985, AJ, 90, 364.

Chapront, J., Chapront-Touzé, M., \& Francou, G. 1999, A\&A, 343, 624

Chapront, J., Chapront-Touzé, M., \& Francou, G. 2000, private communication, to appear in IERS Annual Report

Charlot, P., Sovers, O.J., Williams, J.G., \& Newhall, X.X. 1995, AJ, 109, 418

Dehant, V., et al. 1999, Celest. Mech. Dyn. Astron., 72, 245

Duncombe, R.L., Fricke, W., Seidelmann, P.K., \& Wilkins, G.A. 1977, Trans. IAU, 15B, 56

Fanselow, J.L., Sovers, O.J., Thomas, J.B., Purcell, G.H.Jr., Cohen, E.J., Rogstad, D.H., Skjerve, L.J, \& Spitzmesser, D.J. 1984, AJ, 89, 987

Fukushima, T. 1995, A\&A, 294, 895

Fukushima, T. 2000, Proc. IAU Coll. 180, 417

Groten, E. 2000, Proc. IAU Colloq. 180, 337

Herring, T.A. 1995, Highlights of Astronomy, 10, 222

Herring, T.A., Buffett, B.A., Mathews, P.M., \& Shapiro, I.I. 1991, J. Geophys. Res., 96, 8259

Herring, T.A., Gwinn, C.R., \& Shapiro, I.I. 1986, J. Geophys. Res., 91, 8259

Irwin, A., \& Fukushima, T. 1999, A\&A, 348, 642

Jacobson, R.A., Campbell, J.K., Taylor, A.H., \& Synott, S.P. 1992, AJ, 103, 2068.

Jacobson, R.A., Riedel, J.E., \& Taylor, A.H. 1991, A\&A, 247, 565.

Lieske, J.H., Lederle, T., Fricke, W., \& Morando, B. 1977, A\&A, 58, 1

Mathews, P.M., Buffett, B.A., \& Herring, T.A. 2000, J. Geophys. Res., submitted

McCarthy, D.D. (ed.) 1989, IERS Standards (1989), IERS Tech. Note, 3

McCarthy, D.D. (ed.) 1992, IERS Standards (1992), IERS Tech. Note, 13

McCarthy, D.D. (ed.) 1996, IERS Conventions (1996), IERS Tech. Note, 21 
McCarthy, D.D. (ed.) 2000, IERS Conventions (2000), IERS Tech. Note, in press

McCarthy, D.D., \& Luzum, B.J. 1991, AJ, 102, 1889

Miyamoto, M., \& Soma, M. 1993, AJ, 105, 691

Mohr, P.J., \& Taylor, B.N. 1999 J. Phys. and Chem. Ref. Data, 28, No.6

Null, G.W. 1969, Bull. Amer. Astron. Soc., 1, 356

Petit, G. 2000, Proc. IAU Colloq. 180, 275

Petrov, L. 2000, Proc. IAU Colloq. 180, 254

Roosbeek, F., \& Dehant, V. 1998, Celest. Mech. Dyn. Astron., 70, 215

Seidelmann, P.K. 1982, Celest. Mech., 27, 79

Shirai, T., \& Fukushima, T. 2000, Proc. IAU Colloq. 180, 223

Sjogren, W.L., Trager, G.B., \& Roldan, G.R. 1990, Geophys. Res. Lett., 17, 1485

Souchay, J., Feissel, M., Bizouard, C., Capitaine, N., \& Bougeard, M. 1995, A\&A, 299, 277

Standish, E.M., Jr. 1995, Highlights of Astronomy, 10, 180

Standish, E.M., Jr. 1998, Planetary/Lunar Ephemeris DE405, ftp://navigator.jpl.nasa.gov/ephem/de405.iom

Tholen, D.J., \& Buie, M.W. 1997, Icarus, 125, 245

Vityazev, V. 2000, Proc. IAU Colloq. 180

Vondrák, J. 1999, Celest. Mech. Dyn. Astron., 20, 169

Vondrák, J., \& Ron, C. 2000, Proc. IAU Colloq. 180, 248

Walter, H.G., \& Ma, C. 1994, A\&A, 284, 1000

Walter, H.G., \& Sovers, O.J. 1996, A\&A, 308, 1001

Williams, J.G. 1994, AJ, 108, 711

Wilkins, G.A. 1989, in 'Reference Frames', J. Kovalevsky et al. (eds.), 447

Zhu, S.Y., Groten, E., \& Reigber, Ch. 1990, AJ, 99, 1024 